\title{
THE EUCLIDEAN FACTOR OF A HADAMARD MANIFOLD
}

\author{
TOSHIAKI ADACHI AND FUMIKO OHTSUKA
}

(Communicated by Jonathan M. Rosenberg)

\begin{abstract}
The ideal boundary $X(\infty)$ of a Hadamard manifold $X$ is the set of asymptotic classes of rays on $X$. We shall characterize the Euclidean factor of $X$ by information on $X(\infty)$. Under the assumption that the diameter of $X(\infty)$ is $\pi$, we call a boundary point that has a unique point of Tits distance $\pi$ a polar point. We shall show that such points form a standard sphere and compose the boundary of the Euclidean factor of the given Hadamard manifold.
\end{abstract}

\section{INTRODUCTION}

The purpose of this paper is to make clear the relation between the Euclidean factors of Hadamard manifolds and the shapes of their ideal boundaries. The notion of the ideal boundary $X(\infty)$ of a Hadamard manifold $X$ was introduced in [3] to geometrize some of the basic features of automorphic function theory. With the aid of this notion for visibility manifolds, Eberlein and O'Neill classified them into three types: parabolic, axial, and fuchsian. It is natural and interesting to characterize manifolds by information about their ideal boundaries in several ways. Originally the ideal boundary $X(\infty)$ was defined to be the set of equivalence classes of rays on $X$. The cone and horocycle topologies on $X \cup X(\infty)$ were used for the above classification. Subsequently Gromov [1] defined the Tits metric on $X(\infty)$ and gave us a tool to get further information on $X(\infty)$.

The Tits metric is closely related to flatness or more precisely asymptotical flatness of a Hadamard manifold. For example, $X$ is a visibility manifold if and only if $X(\infty)$ is discrete with respect to the Tits topology and $X$ is Euclidean if and only if $X(\infty)$ is isometric to a standard sphere. Furthermore if $X$ contains a $k$-dimensional Euclidean factor, then a $(k-1)$-dimensional standard sphere is isometrically embedded in $X(\infty)$. The opposite of this statement is not true in general: When $X(\infty)$ contains a standard sphere, then either $X$ contains a corresponding flat or $X(\infty)$ contains a hemisphere whose boundary is the given

Received by the editors April 4, 1990 and, in revised form, September 24, 1990.

1980 Mathematics Subject Classification (1985 Revision). Primary 53C99.

Key words and phrases. Euclidean factor, Tits metric, polar point, Hadamard manifold.

The first author was partially supported by Grant-in-Aid for Encouragement of Young Scientists (No.02740028), The Ministry of Education, Science and Culture, Japan.

The second author was partially supported by Grant-in-Aid for Encouragement of Young Scientists (No.01740011), The Ministry of Education, Science and Culture, Japan. 
sphere (see p. 229 [1]). Hence $X$ does not necessarily have a corresponding Euclidean factor.

The second author [5] pointed out that $X$ splits as $X^{\prime} \times \mathbf{R}$ if the diameter of $X(\infty)$ is $\pi$ and there exists a point $N \in X(\infty)$ satisfying the following condition;

(*) there exists only one point $S \in X(\infty)$ with $T d(N, S)=\pi$,

where $T d$ denotes the Tits distance (for the definition, see $\S 1$ ). In the case that $X=X^{\prime} \times \mathbf{R}^{k}$, every point of the ideal boundary $\mathbf{R}^{k}(\infty)$ of the Euclidean factor satisfies this condition $(*)$. In this note we pay attention to such points, which we call polar points, in order to characterize the Euclidean factor of $X$ and show that the set of polar points, if nonempty, forms an isometrically embedded standard sphere and composes the ideal boundary of the Euclidean factor of $X$. This assures that we can determine the Euclidean factors of Hadamard manifolds just by looking at their ideal boundaries.

\section{Polar Points}

In this section we shall prepare some notations and basic properties of polar points.

Let $X$ be a Hadamard manifold, which is a simply connected complete Riemannian manifold of nonpositive curvature. We always suppose that geodesics are parametrized by arc length. Two geodesic rays $\gamma_{1}, \gamma_{2}:[0, \infty) \rightarrow X$ are said to be asymptotic if the distance function $t \rightarrow d\left(\gamma_{1}(t), \gamma_{2}(t)\right)$ is bounded for $t \geq 0$. This asymptotic relation is an equivalence relation. The ideal boundary $X(\infty)$ of $X$ is the set of all asymptotic classes of geodesic rays in $X$. The Tits metric on this boundary is defined in the following manner. Given points $x \in X$ and $z \in X(\infty)$ we have the geodesic ray $\gamma_{x z}$ in $X$ emanating from $x$ whose asymptotic class $\gamma_{x z}(\infty)$ is $z$. One can define the angle metric $\angle(z, w)$ on $X(\infty)$ as the supremum of the angle $L_{x}(z, w)=\angle\left(\dot{\gamma}_{x z}(0), \dot{\gamma}_{x w}(0)\right)$. The Tits metric $T d(\cdot, \cdot)$ is the interior metric induced from this angle metric. For general information on the Tits metric see $\S 4$ of [1].

We here recall the following concept which is useful in our argument. Two totally geodesic submanifolds $Y_{1}$ and $Y_{2}$ of $X$ are called parallel if the Hausdorff distance between them is finite. For example, two geodesics $c_{1}, c_{2}$ are parallel if and only if $\left\{c_{1}(\infty), c_{1}(-\infty)\right\}=\left\{c_{2}(\infty), c_{2}(-\infty)\right\}$, where $c_{i}(\infty)$ and $c_{i}(-\infty)$ denote the asymptotic classes containing the rays $c_{i}(t), c_{i}(-t):[0, \infty) \rightarrow X$, respectively. The union of all totally geodesic submanifolds parallel to a totally geodesic submanifold $Y$ is isometric to $Y \times Z$, where $Z$ is a closed convex subset of $X$ (see Lemma 2.4 [1]).

From now on we suppose the diameter of the ideal boundary $X(\infty)$ of $X$ to be equal to $\pi$. This happens if and only if every geodesic in $X$ bounds a flat half plane (see Lemma 4.10 [1]). In particular a Hadamard manifold has no Euclidean factor if the diameter of the ideal boundary is greater than $\pi$. Hence this assumption does not impose any restrictions. 
We shall call a point $N$ in $X(\infty)$ a polar point if there exists only one point $S$ with $T d(N, S)=\pi$ (cf. p. 77 [2]). This condition guarantees that a geodesic $c$ with $c(\infty)=N$ satisfies $c(-\infty)=S$. Thus all geodesics belonging to $N$ are parallel and $X$ splits as $X^{\prime} \times \mathbf{R}$ with $\mathbf{R}(\infty)=\{N, S\}$. For points $z \in X(\infty)$ and $x \in X$ the geodesics $\gamma_{x N}$ and $\gamma_{x z}$ span a flat totally geodesic plane in $X$, since $\gamma_{x N}$ is tangent to the R-factor of $X$. Hence we get the following equality:

$$
T d(N, z)=\angle(N, z)=L_{x}\left(\dot{\gamma}_{x N}(0), \dot{\gamma}_{x z}(0)\right) \text {. }
$$

We simultaneously have that $T d(N, z)+T d(z, S)=\pi$ and that $S$ is also polar. We hence call $S$ the opposite polar point of $N$.

We now consider the case that there are two polar points $N_{1}$ and $N_{2}$ with $T d\left(N_{1}, N_{2}\right)<\pi$ (i.e. one is not the opposite polar point of the other). Given a point $x \in X$, let $c_{1}, c_{2}$ be geodesics such that $c_{1}(\infty)=N_{1}, c_{2}(\infty)=N_{2}$, and $c_{1}(0)=c_{2}(0)=x$. By the equality $\angle\left(N_{1}, N_{2}\right)=L_{x}\left(\dot{c}_{1}(0), \dot{c}_{2}(0)\right)<\pi$, we can conclude the geodesic rays $c_{1}, c_{2}:[0, \infty) \rightarrow X$ bound a totally geodesic Euclidean sector (see p. 34 [1]). By the same arguments we see that three other segments spanned by $c_{1}, c_{2}$ are flat and together span a flat plane $E_{x}$. Since $N_{1}$ and $N_{2}$ are polar, the boundary $E_{x}(\infty)$ consists of four Tits geodesics of length smaller than $\pi$ whose endpoints lie in the set $\left\{N_{1}, N_{2}, S_{1}, S_{2}\right\}$, where $S_{1}, S_{2}$ are the polar points opposite to $N_{1}, N_{2}$. The uniqueness of Tits geodesics of length smaller than $\pi$ implies that $E_{x}(\infty)$ does not depend on the choice of $x$. Therefore the Hausdorff distance between two planes $E_{x}$ and $E_{y}$ is not greater than $d(x, y)$. Hence $E_{x}$ all are parallel, and we get the following.

Lemma. If there are polar points $N_{1}$ and $N_{2}$ such that $N_{2}$ is not the opposite polar point of $N_{1}$, then there exists an isometrically embedded circle $S^{1}$ in $X(\infty)$ containing $N_{1}$ and $N_{2}$, and $X$ splits as $X^{\prime} \times \mathbf{R}^{2}$ so that $\mathbf{R}^{2}(\infty)=S^{1}$.

This implies that every point on $S^{1}$ in $X(\infty)$ is polar and leads us to the following corollary.

Corollary. Polar points form a standard sphere.

Proof. Let $N_{1}, \ldots, N_{k}$ be polar points. For a point $x \in X$, let $\gamma_{x j}$ be the ray emanating from $x$ such that $\gamma_{x j}(\infty)=N_{j}$ for $j=1, \ldots, k$. Applying the above lemma inductively, we can conclude that $c(\infty)$ is also polar if the initial velocity vector $\dot{c}(0)$ of a geodesic $c$ is contained in the linear subspace of $T_{x} X$ spanned by the vectors $\dot{\gamma}_{x j}(0)$. In other words, the set $S_{x}$ of vectors $\dot{c}(0)$ of geodesics $c$ such that $c(\infty)$ is a polar point and $c(0)=x$ is a standard sphere in the unit tangent sphere $T_{x}^{1} X$. Since we have by the equality $(* *)$ that the natural map between $\left(T_{x}^{1} X, \angle\right)$ onto $(X(\infty), T d)$ is isometric when restricted to $S_{x}$, we get the assertion.

\section{EUCLIDEAN RANK OF AN IDEAL BOUNDARY}

We now show that polar points as studied in the previous section are closely related to the Euclidean factor of $X$. We say that the ideal boundary $X(\infty)$ 
of a Hadamard manifold $X$ has Euclidean rank $k$ if the set of all polar points forms a $(k-1)$-dimensional standard sphere $S^{k-1}$. Particularly we say that the Euclidean rank is 0 if $X(\infty)$ does not contain any polar points. This means that if the diameter of $X(\infty)$ is greater than $\pi$, then the Euclidean rank of $X$ is 0 . If $X=X^{\prime} \times \mathbf{R}^{k}$, then every point of $\mathbf{R}(\infty)=S^{k-1}$ is polar, and the Euclidean rank of $X$ is equal to or greater than $k$. Furthermore we have the following.

Theorem. The Euclidean rank of $X(\infty)$ coincides with the dimension of the Euclidean factor of $X$.

Proof. Let $k$ be the Euclidean rank of $X(\infty)$ and $S^{k-1}$ be the set of all polar points. For $x \in X$, we define a subset $F_{x}$ of $X$ by

$$
\bigcup\left\{c(\mathbf{R}) \mid c: \mathbf{R} \rightarrow X \text { is a geodesic with } c(0)=x \text { and } \dot{c}(0) \in S^{k-1}\right\} .
$$

Given two distinct points $y_{1}$ and $y_{2}$ of $F_{x}$, we get that $x, y_{1}$, and $y_{2}$ lie in a flat totally geodesic embedded 2-plane in $F_{x}$. Hence $F_{x}$ contains the unique maximal geodesic joining $y_{1}$ and $y_{2}$, and it follows that $F_{x}$ is a complete totally geodesic submanifold of $X$. Since the ideal boundary $F_{x}(\infty)$ of the Hadamard manifold $F_{x}$ is isometric to a standard sphere with respect to the Tits metric for $F_{x}$, we can conclude that $F_{x}$ is flat . Moreover $F_{x}(x \in X)$ all are parallel because their boundaries coincide. We therefore get $X$ splits as $X_{0} \times F$, where $F$ is isometric to $F_{x}$, hence is the $k$-dimensional Euclidean space. Here if $X_{0}$ has a Euclidean factor, then $X(\infty)=\left(X_{0}^{\prime} \times \mathbf{R} \times \mathbf{R}^{k}\right)(\infty)$, and the Euclidean rank of $X$ is not smaller than $k+1$, which is a contradiction. Hence $X_{0}$ has no Euclidean factor. This completes the proof of the theorem.

Remark. An isometry between ideal boundaries maps polar points to polar points. We therefore get the following: If the ideal boundaries of two Hadamard manifolds are isometric then their Euclidean factors coincide.

\section{REFERENCES}

1. W. Ballmann, M. Gromov, and V. Schroeder, Manifolds of nonpositive curvature, Progr. Math., vol. 61, Birkhäuser, Basel, 1985.

2. S. Chen and P. Eberlein, Isometry groups of simply connected manifolds of nonpositive curvature, Illinois J. Math. 24 (1980), 73-103.

3. P. Eberlein and B. O'Neill, Visibility manifolds, Pacific J. Math. 46 (1973), 45-109.

4. F. Ohtsuka, On a relation between total curvature and Tits metric, Bull. Fac. Sci. Ibaraki Univ. Ser. A 20 (1988), 5-8.

5. __ On manifolds having some restricted ideal boundaries, Geom. Dedicata (to appear).

Department of Mathematics, Nagoya Institute of Technology, Showa-ku, Nagoya 466, JAPAN

E-mail address: d43019a@nucc.cc.nagoya-u.ac.jp

Department of Mathematics, Faculty of Science, Ibaraki University, Mito 310, Japan 